\title{
Atividades de enfermagem ao paciente prostatectomizado
}

\author{
Nursing prostatectomy patients \\ Actividades de enfermería al paciente prostatectomizado
}

\author{
Daniele Raiane Florentino dos Santos I Camila Araújo Florêncio de Lima ${ }^{\text {II }}$ Elisandra de Araújo SaldanhalII; Maria Isabel da \\ Conceição Dias Fernandes Cavalcanti ${ }^{V I}$; Ana Beatriz de Almeida Medeiros ${ }^{V}$; Ana Luisa Brandão de Carvalho Lira ${ }^{\mathrm{VI}}$
}

\begin{abstract}
RESUMO: Estudo descritivo, cujo objetivo foi identificar as atividades realizadas pelos profissionais de enfermagem da urologia no cuidado ao paciente em pós-operatório de prostatectomia e compará-las ao apresentado na literatura. A pesquisa foi realizada em um hospital universitário, localizado na Região Nordeste do Brasil, nos meses de agosto e setembro de 2011, com 18 profissionais de enfermagem, incluindo enfermeiros, técnicos de enfermagem e auxiliares de enfermagem, que responderam a um questionário contendo perguntas sobre seus dados sociodemográficos e sobre os cuidados prestados pela enfermagem ao paciente em pós-operatório de prostatectomia. Os cuidados de enfermagem foram agrupados em seis categorias: orientações sobre o período pós-operatório; tratamento da incontinência urinária; tratamento da disfunção erétil; tratamento da dor; acompanhamento psicológico; e tratamento da hiponatremia. Conclui-se que os profissionais de enfermagem enfatizaram as orientações no pós-operatório, o tratamento da incontinência urinária e da dor e o acompanhamento psicológico, em descompasso com a literatura.
\end{abstract}

Palavras-Chave: Cuidados de enfermagem; prostatectomia; período pós-operatório; enfermagem prática.

ABSTRACT: This descriptive study aimed to identify the activities of urological nursing professionals in caring for postoperative prostatectomy patients, and to compare them with those described in the literature. It was conducted at a university hospital in northeastern Brazil, in August and September 2011, with 18 nursing professionals, including nurses, nurse technicians and nursing assistants, who answered a questionnaire about their sociodemographic data and the care they provided to postoperative prostatectomy patients. The nursing care was grouped into six categories: postoperative guidance; treatment of urinary incontinence; treatment of erectile dysfunction; treatment of pain; psychological monitoring; and treatment of hyponatremia. It was concluded that nursing professionals, at odds with the literature, emphasized postoperative guidance, treatment of urinary incontinence and pain, and mental health counseling.

Keywords: Nursing care; prostatectomy; postoperative period; practical nursing.

RESUMEN: Estudio descriptivo, cuyo objetivo fue identificar las actividades realizadas por los profesionales de enfermería de urología en la atención al paciente en el postoperatorio de prostatectomía y compararlas con lo que se presenta la literatura. Se llevó a cabo en un hospital universitario del noreste de Brasil, en los meses de agosto y septiembre de 2011, junto a 18 profesionales de enfermería, incluyendo enfermeros, técnicos de enfermería y auxiliares de enfermería, que respondieron a un cuestionario sobre sus datos sociodemográficos y sobre los cuidados prestados por la enfermería al paciente en postoperatorio de prostatectomía. Los cuidados de enfermería se agruparon en seis categorías: orientación sobre el postoperatorio; tratamiento de la incontinencia urinaria; tratamiento de la disfunción eréctil; tratamiento del dolor; seguimiento psicológico; y tratamiento de la hiponatremia. Se concluyó que los profesionales de enfermería ponen relieve las orientaciones en postoperatorio, el tratamiento de la incontinencia urinaria y del dolor y el seguimiento psicológico, diferentemente de lo que expone la literatura. Palabras Clave: Atención de enfermería; prostatectomía; periodo postoperatorio; enfermería práctica.

\section{INTRODUÇÃO}

Mundialmente, as doenças relacionadas à próstata, principalmente o câncer e a Hiperplasia Benigna da Próstata (HBP), têm sido discutidas como um relevante problema de saúde pública. No Brasil, a elevação dos índices de incidência dessas doenças ao longo dos anos ocorreu devido ao aumento da expectativa de vida populacional, à evolução das técnicas de diagnóstico e ao progresso dos sistemas de informação do país ${ }^{1}$.

O câncer de próstata é apontado como o segundo tipo de câncer mais frequente em homens, com taxa de incidência mundial crescente ${ }^{1}$. Já a HBP é considerada

'Enfermeira. Universidade Federal do Rio Grande do Norte. Natal/Rio Grande do Norte, Brasil. E-mail: daniele_raiane@hotmail.com.

"IEnfermeira. Universidade Federal do Rio Grande do Norte. Natal/Rio Grande do Norte, Brasil. E-mail: camillabecker@@hotmail.com.

IIIEnfermeira. Mestre em Enfermagem. Universidade Federal do Rio Grande do Norte. Natal/Rio Grande do Norte, Brasil. E-mail: sansaldanha@yahoo.com.br.

IVEnfermeira. Doutoranda em Enfermagem. Universidade Federal do Rio Grande do Norte. Natal/Rio Grande do Norte, Brasil. E-mail: bebel_6@ hotmail.com.

vEnfermeira. Doutoranda em Enfermagem. Universidade Federal do Rio Grande do Norte. Natal/Rio Grande do Norte, Brasil. E-mail: abamedeiros@gmail.com.

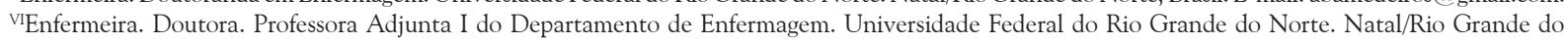

Norte, Brasil. E-mail: analuisa_brandao@yahoo.com.br. 
uma das três doenças que mais contribuem para as despesas de saúde nos países industrializados. Além disso, de cada 100 mil homens, 30 morrem por causa da HBP²

O tratamento para ambas as doenças depende do quadro clínico do paciente. As opções vão desde a conduta conservadora, como a observação e o tratamento medicamentoso, até os procedimentos cirúrgicos, os quais podem trazer diversas complicações, acometendo tanto o aspecto físico como psicológico do indivíduo ${ }^{3}$.

Dentre as complicações, destacam-se a incontinência urinária, a disfunção erétil, a dor e a formação de coágulos que podem obstruir o cateter vesical de demora, distender a cápsula prostática e provocar hemorragia. Ademais, após a cirurgia, o paciente também pode vivenciar conflitos emocionais, dentre esses o medo, o desamparo, a raiva e a tristeza. Esses sentimentos podem ser intensificados pelas expectativas criadas com relação à possibilidade de curar-se de uma doença grave e por dúvidas referentes ao autocuidado ${ }^{4}$.

Nesse contexto, torna-se fundamental o papel da equipe de enfermagem, que deve prestar cuidados voltados ao aspecto físico do indivíduo, além de fornecer apoio e orientações adequadas para amenizar as incertezas e os possíveis efeitos colaterais do procedimento ${ }^{4}$.

Assim, a enfermagem deve orientar esse paciente quanto aos cuidados pós-cirúrgicos relacionados à sonda vesical de demora, tais como banhos mornos, lavagem da ponta do pênis com frequência, utilização de pomada e roupas folgadas. Deve explicar, ainda, sobre o tempo de recuperação, a evolução desse período e alertar para as possíveis complicações da cirurgia, como a incontinência urinária e a disfunção erétil ${ }^{5}$. Ademais, a família e/ou os cuidadores do paciente devem ser incluídos nessas orientações sobre o autocuidado ${ }^{6}$.

Desse modo, em vista do crescente número de indivíduos submetidos à cirurgia de próstata e da necessidade de uma assistência individualizada e peculiar, torna-se fundamental o conhecimento acerca dos cuidados prestados pela equipe de enfermagem aos pacientes em pós-operatório de prostatectomia. Tal conhecimento poderá aprimorar a conduta dos profissionais de enfermagem e orientá-los a realizar um cuidado de maior qualidade, capaz de atender às necessidades desta população em um período que demanda atenção bastante singular.

Com base no exposto, surgiu o seguinte questionamento: Os cuidados realizados pela equipe de enfermagem ao paciente prostatectomizado de um hospital universitário de uma cidade do Nordeste do Brasil estão de acordo com os recomendados pela literatura?

A fim de contemplar tal questionamento, objetivou-se, nesta pesquisa, identificar as atividades executadas pelos profissionais de enfermagem da urologia no cuidado ao paciente em pós-operatório de prostatectomia e compará-las ao que é apresentado na literatura.

\section{REVISÃo DE LITERATURA}

A cirurgia prostática é comumente indicada aos pacientes com HBP ou com câncer de próstata. Dentre as condutas cirúrgicas adotadas no tratamento dos distúrbios dessa glândula, destacam-se a ressecção transuretral (RTUP), a incisão transuretral (ITUP), a prostatectomia radical laparoscópica e a remoção cirúrgica aberta, a qual possui três diferentes modalidades: a suprapúbica, a perineal e a retropúbica ${ }^{3}$.

A RTUP é o procedimento geralmente utilizado, considerado o padrão-ouro, sendo realizado por meio da endoscopia, não requerendo, portanto, incisão. Para tanto, introduz-se um instrumento óptico através da uretra até a próstata e remove-se a glândula em pequenas porções. Algumas das potenciais complicações deste procedimento são a disfunção erétil, a ejaculação retrógrada e a síndrome da ressecção transuretral, que, embora ocorra em 2\% dos homens que se submetem a esse tratamento, pode provocar letargia, confusão, hipotensão, taquicardia, náusea, vômito, cefaleia, espasmos musculares e convulsões ${ }^{3,7}$.

A ITUP, assim como a RTUP, é realizada por meio de um instrumento introduzido na uretra até a próstata, onde são feitas duas incisões com vistas a reduzir a pressão prostática sobre a uretra. É um procedimento realizado em ambulatório e é caracterizado por apresentar um índice de complicações menor do que os demais procedimentos ${ }^{3}$.

A prostatectomia radical laparoscópica é considerada um procedimento minimamente invasivo, em que os pacientes apresentam menor sangramento, internações mais curtas e retornam mais rapidamente às suas atividades habituais. Entretanto, devido à incapacidade do cirurgião de palpar a próstata no momento da cirurgia, pode haver comprometimento dos nervos cavernosos, o que pode causar disfunção erétil ${ }^{3,8}$.

Na remoção cirúrgica aberta, a próstata é retirada completamente. Para isso, existem três diferentes métodos, a saber: a prostatectomia suprapúbica, perineal e retropúbica. Essas condutas diferem umas das outras no que tange à localização da incisão e das complicações pós-cirúrgicas ${ }^{3}$.

No primeiro método, faz-se uma incisão diretamente na bexiga para a retirada da glândula. Como complicação, pode ocorrer hemorragia de difícil controle. No segundo tipo, a incisão é na região perineal, o que aumenta as chances de infecção, já que a incisão é próxima ao reto, podendo ocorrer, também, a incontinência, impotência e lesão retal. Na retropúbica, o cirurgião retira a próstata entre o arco púbico e a bexiga, sem penetrá-la. Como complicação, pode ocorrer a infecção precoce no espaço retropúbico ${ }^{3}$.

Somados às complicações relacionadas aos procedimentos cirúrgicos, existem ainda os conflitos emocionais com os quais o paciente poderá conviver 
após a cirurgia, como o medo, o desamparo, a raiva e a tristeza. Esses sentimentos possuem íntima relação com as expectativas e dúvidas advindas da eficácia da terapia e os possíveis efeitos colaterais existentes ${ }^{4}$.

Portanto, tendo-se em vista a ocorrência dos conflitos emocionais e as possíveis complicações durante o pós-operatório de prostatectomia, a equipe de enfermagem deverá traçar cuidados pertinentes a essa clientela, no intuito de minimizar essas complicações e promover o restabelecimento da saúde física e psicológica dos pacientes submetidos a esse tratamento.

Para tanto, segundo a literatura, o enfermeiro deverá prestar como cuidados: acompanhamento psicológico, de forma a identificar as necessidades dos pacientes, fornecendo apoio e/ou encaminhando para outros setores mais especializados; fornecer orientações sobre o pós-operatório, como dar orientações sobre a recuperação cirúrgica, os cuidados pós-cirúrgicos e suas eventuais complicações; tratamento da disfunção erétil e da incontinência, da dor e da hiponatremia9.

\section{Metodologia}

Trata-se de um estudo descritivo, realizado em um hospital universitário localizado em uma cidade do Nordeste do Brasil. Esse é um hospital público de grande porte, prestador de serviços ao Sistema Único de Saúde (SUS), sendo uma referência de média e alta complexidades para todo o estado. Comporta 191 leitos de internação, distribuídos nos setores de unidade de terapia intensiva (UTI), transplante e enfermarias.

A população do estudo foi constituída por todos os profissionais de enfermagem (enfermeiros, técnicos de enfermagem e auxiliares de enfermagem) do hospital. A amostra foi composta pelos profissionais de enfermagem que trabalhavam nas enfermarias de urologia do hospital, totalizando 18 profissionais reunidos por meio de amostragem consecutiva.

Os critérios de inclusão foram: trabalhar na assistência de enfermagem ao paciente prostatectomizado e estar na escala nos meses da coleta dos dados. Determinou-se como critérios de exclusão a discordância dos critérios de inclusão anteriormente citados.

Para a coleta dos dados, empregou-se um questionário semiestruturado, com perguntas abertas e fechadas relativas aos dados sociodemográficos dos profissionais e aos cuidados prestados aos pacientes em pós-operatório de prostatectomia. Para tanto, foram utilizadas as variáveis: idade, sexo, formação profissional, anos de exercício como profissional de enfermagem e como atuante na unidade de urologia e se o profissional havia recebido algum treinamento.

As demais variáveis do questionário foram as categorias referentes aos cuidados de enfermagem prestados no pós-operatório de prostatectomia, preestabeleci- das pelos pesquisadores, por meio de busca literária prévia. As categorias referentes aos cuidados foram as seguintes: orientações sobre o período pós-operatório; tratamento da incontinência urinária; tratamento da disfunção erétil; tratamento da dor; acompanhamento psicológico; e tratamento da hiponatremia.

Para tanto, os profissionais de enfermagem eram indagados sobre a realização de algum desses cuidados; caso a resposta fosse positiva, as atividades para cada categoria de cuidados eram descritas por extenso. Os dados foram coletados nos meses de agosto e setembro de 2011.

As atividades de enfermagem relatadas pelos profissionais foram agrupadas por semelhança de palavras e significados dos termos e, então, apresentadas em número absoluto de citações. Por conseguinte, tais atividades foram comparadas aos cuidados de enfermagem evidenciados pela literatura, buscando identificar palavras diferentes com significados semelhantes, método utilizado em estudo similar ${ }^{10}$. Esses termos foram, posteriormente, reunidos por semelhança de conteúdo e agrupados nas seis categorias previamente estabelecidas. Destaca-se que, para cada categoria, foram calculados os números absoluto e relativo das citações de cada cuidado de enfermagem prestado pelo profissional. Para tanto, os dados foram submetidos à análise estatística descritiva, sendo expostas as frequências relativa e absoluta, podendo haver respostas múltiplas.

Nesta pesquisa, respeitaram-se os preceitos éticos e legais a serem seguidos nas investigações envolvendo seres humanos. $\mathrm{O}$ estudo foi apreciado pelo Comitê de Ética em Pesquisa do referido hospital, que o aprovou com número de protocolo 590/11 e com Certificado de Apresentação para Apreciação Ética (CAAE) 0031.0.294.000-11.

\section{Resultados e Discussão}

No tocante à caracterização sociodemográfica dos participantes da pesquisa, a faixa etária variou entre 20 e 57 anos, sendo a idade de 41 anos a mais frequente, representada por $3(16,6 \%)$ profissionais. Os $18(100 \%)$ profissionais eram do sexo feminino e a maioria deles, $11(61,1 \%)$, atuava na instituição como técnico de enfermagem. Os profissionais de enfermagem de nível médio compõem um número expressivo e representativo nas instituições de saúde, sendo, portanto, responsáveis por inúmeros cuidados aos pacientes ${ }^{11}$.

O tempo de serviço como profissional de enfermagem variou de 41 dias a 28 anos, prevalecendo a faixa de um ano, referente a $3(16,6 \%)$ pessoas. Como profissional da unidade de urologia, o tempo variou de 15 dias a 10 anos. A maioria dos participantes, $17(94,4 \%)$, não recebeu treinamento em serviço. 
Na primeira categoria, orientações sobre o período pós-operatório, os profissionais de enfermagem relataram 15 cuidados, sendo o repouso a principal orientação descrita por eles. Em contraponto, entre os 15 cuidados citados, somente 3(16,6\%) estão em consonância com a literatura, a saber: esclarecimento sobre as complicações do pós-operatório, citado por apenas três profissionais, incentivar a ingesta de líquidos e cuidados com a sonda vesical de demora, citados por apenas dois profissionais investigados.

A cirurgia de próstata gera muitas dúvidas e questões complexas para o paciente e sua família, pois envolve aspectos funcionais, urológicos e sexuais. Portanto, a equipe de saúde, principalmente a de enfermagem, deve conduzir métodos de orientação e comunicação para facilitar o restabelecimento desse paciente. Assim, as orientações sobre o período pós-operatório para o paciente e o seu acompanhante (cônjuge/cuidador) são de suma importância para uma recuperação adequada 9 .

Ademais, o período pós-operatório da prostatectomia é, por vezes, difícil de ser vivenciado, pois o paciente pode apresentar complicações como infecção, bloqueio do tecido cicatricial, espasmos da bexiga, dor, incontinência urinária e disfunção sexual. Assim, ele e seu cônjuge/cuidador devem ser claramente informados, pois geralmente estão concentrados somente no procedimento cirúrgico que representa a esperança da cura, sem atentar para o que virá posteriormente ${ }^{12}$.

Com relação à ingesta de líquidos, em especial de água, se for ingerida em quantidade adequada, previne a formação de coágulos sanguíneos que, quando presentes, podem obstruir o cateter vesical e causar dor. Assim, o paciente deve ser estimulado a aumentar a ingesta hídrica, principalmente no primeiro mês após a cirurgia e enquanto houver hematúria ${ }^{13}$. Além disso, a ingesta de água adequada evita a desidratação, problema de enfermagem relatado por estudo de caso com paciente prostatectomizado ${ }^{14}$.

É comum a todos os pacientes prostatectomizados o uso da sonda vesical de demora (SVD) nas primeiras semanas de pós-operatório. Esse cateter, apesar de trazer benefícios, pode causar danos físicos e emocionais, como infecção do trato urinário (ITU), raiva e frustração. Assim, a enfermagem deve explicar como o paciente e seu cuidador devem lidar com a SVD e ajudá-los a aceitá-la ${ }^{13}$.

Na segunda categoria - tratamento da incontinência urinária -, a principal atividade dos profissionais foi proporcionar ao paciente o uso de fraldas. Entretanto, esse tipo de atividade não atua diretamente na causa do problema, é apenas um paliativo. Além disso, em estudo sobre autopercepção de idosos sobre o uso de fraldas quando hospitalizados demonstrou, como principais sentimentos relacionados ao seu uso, o incômodo, alteração na autoestima e aversão à fralda. Nesse sentido, como conclusão, o estudo assevera que o uso de fraldas envolve não apenas questões relacionadas à necessidade de sua utilização, mas também questionamentos a respeito de como a utilização dessas refletirá fisicamente e emocionalmente na vida de quem as utiliza ${ }^{15}$.

Nesse aspecto, a equipe de enfermagem deste estudo deverá refletir e relacionar melhor suas condutas ao perfil dos clientes estudados. É de suma importância avaliar com melhor exatidão o contexto da clientela e a existência de outras técnicas que possam ser utilizadas para essa problemática, considerando as questões de autoestima a ela inerentes.

Destarte, o cuidado relativo à realização de exercícios perineais, encontrado na literatura como uma forma de tratamento para o problema em questão, foi relatado somente por um profissional de enfermagem. Esses exercícios são um meio não invasivo de corrigir a incontinência urinária que afeta diretamente a qualidade de vida dos indivíduos prostatectomizados ${ }^{16}$. A literatura afirma que tais exercícios são, de fato, a opção mais eficiente para o tratamento da incontinência, pois apresentam resultados positivos na prática ${ }^{17}$.

As categorias tratamento da disfunção erétil, na qual foi relatado apenas um cuidado, e tratamento da dor, em que o principal cuidado de enfermagem foi a administração de medicamentos conforme prescrição médica, são apresentados na Tabela 1 .

TABELA 1: Atividades de enfermagem/cuidado: tratamento da disfunção erétil e tratamento da dor em pacientes prostatectomizados. Natal/RN, 2011.

\begin{tabular}{llc}
\hline Categorias & Atividades/cuidado de enfermagem & $f(\%)$ \\
\hline Tratamento da disfunção erétil & 1. Encaminhar o paciente ao profissional especialista & $3(16,6)$ \\
Tratamento da dor & 1. Administrar medicamentos conforme prescrição médica & $15(83,3)$ \\
& 2. Localizar a dor do paciente & $2(11,1)$ \\
& 3. Evitar esforço físico & $1(5,5)$ \\
& 4. Orientar o repouso & $1(5,5)$ \\
& 5. Verificar a presença de coágulo na SVD & $1(5,5)$ \\
& 6. Instilar soro na SVD & $1(5,5)$ \\
7. Informar ao médico a presença da dor & $1(5,5)$ \\
8. Solicitar o aviso do paciente, se houver dor & $1(5,5)$ \\
9. Aplicar compressa no local da dor & $1(5,5)$ \\
\hline
\end{tabular}


Na categoria tratamento da disfunção erétil, o único cuidado de enfermagem citado foi encaminhar o paciente ao profissional especialista. A disfunção erétil é comum em pacientes prostatectomizados, afetando pelo menos metade dos pacientes com câncer de próstata. Esse problema ocorre, geralmente, em virtude de danos aos nervos e vasos sanguíneos envoltos da próstata. Entre as consequências desse dano físico, identificamse a depressão, ansiedade, conflito de relacionamento e perda da autoestima ${ }^{18}$. Por isso, a enfermagem deve intervir para a resolução desse problema, não somente encaminhando o paciente a outro profissional, mas assumindo sua autonomia de intervir.

Nessa situação, o profissional de enfermagem pode se mostrar disponível para o esclarecimento de dúvidas; explicar a natureza da disfunção erétil; orientar sobre possíveis tratamentos para o problema, como o uso de inibidores da fosfodiesterase, supositório intrauretral, injeção peniana, dispositivo a vácuo e prótese peniana; incentivar diferentes formas de satisfação entre o casal, como, por exemplo, aumentar a expressão do afeto sem exigir o contato sexual ${ }^{9}$.

A categoria tratamento da dor apresentou nove cuidados citados pelos profissionais participantes da pesquisa. Desses, somente um foi comum à literatura: administração de medicamentos conforme prescrição médica, conforme relatado por 15 (83,3\%) participantes. Segundo a literatura, deve-se administrar opioides e/ou anti-inflamatórios não esteroides. Entretanto, poucos estudos abordam o tratamento da dor como uma atividade de enfermagem, indicando que esses profissionais podem não estar considerando significativa essa alteração tão comum presente no pós-operatório ${ }^{13,19}$. Em contraponto, a dor é um importante diagnóstico de enfermagem, o qual é comumente identificado em pacientes submetidos ao pós-operatório, podendo repercutir nos âmbitos psicológico e fisiológico quando não sanado ${ }^{14}$.

As categorias acompanhamento psicológico, tratamento da hiponatremia e os seus respectivos cuidados estão descritas na Tabela 2.
A categoria acompanhamento psicológico apresentou seis cuidados, sendo o mais relatado apoiar o paciente e o seu acompanhante, por $10(55,5 \%)$ participantes. Entre os cuidados, apenas um não foi citado pela literatura: o técnico de enfermagem deve comunicar ao enfermeiro a necessidade do paciente. Tal achado pode estar relacionado à pouca distinção entre as diferentes categorias de profissionais de enfermagem.

Estudo sobre o impacto dos cuidados de enfermagem em homens após tratamento da próstata indicou que $81 \%$ dos entrevistados possuíam alguma necessidade de cuidado não satisfeita, e, dentre essas, incluía-se, principalmente, o suporte à saúde psicológica, sexual e orientações relacionadas ${ }^{20}$. Assim, a enfermagem pode identificar e discutir as necessidades de suporte psicológico para o paciente, ouvir suas angústias e necessidades, fornecer apoio emocional, aconselhamento ou encaminhamento para outros recursos e incentivar a sua participação em grupos de apoio? .

Em revisão sistemática sobre como o acompanhamento telefônico a pacientes após prostatectomia pode influenciar na melhoria dos efeitos clínicos e psicológicos, identificou-se que esse tipo de acompanhamento gera efeitos benéficos ao paciente, sendo, portanto, uma ferramenta recomendada aos enfermeiros, com vistas a melhorar a qualidade de vida dos pacientes vivenciando esse contexto ${ }^{21}$.

$\mathrm{Na}$ segunda categoria relacionada ao tratamento da hiponatremia, $5(27,7 \%)$ profissionais relataram como cuidado de enfermagem a necessidade de se administrar eletrólitos por via endovenosa conforme prescrição médica. Entretanto, nessa categoria, apenas três atividades foram citadas pelos profissionais, sendo, portanto, identificado o relato de poucas atividades para o tratamento da hiponatremia, as quais foram encontradas em apenas um estudo na literatura ${ }^{22}$.

Entretanto, a hiponatremia é uma complicação que pode ocorrer no pós-operatório de prostatectomia e, portanto, a equipe de enfermagem deve estar apta a conhecer a fisiopatologia e os sinais e sintomas dessa

TABELA 2: Atividades de enfermagem/cuidado: acompanhamento psicológico e tratamento da hiponatremia em pacientes prostatectomizados. Natal/RN, 2011.

\begin{tabular}{llc}
\hline Categorias & Atividades/cuidado de enfermagem & $\mathbf{f ( \% )}$ \\
\hline Acompanhamento & 1. Apoiar o paciente e seu acompanhante & $10(55,5)$ \\
psicológico & 2. Conversar e ouvir o paciente & $9(50)$ \\
& 3. Encaminhar o paciente ao psicólogo se necessário & $3(16,6)$ \\
& 4. Orientar a família a acalmar o paciente & $3(16,6)$ \\
& 5. Proporcionar conforto ao paciente & $1(5,5)$ \\
& 6. O técnico de enfermagem deve comunicar ao enfermeiro a necessidade & $1(5,5)$ \\
Tratamento da & \multicolumn{1}{c}{ do paciente } & $5(27,7)$ \\
hiponatremia & 1. Administrar eletrólitos por via endovenosa conforme prescrição médica & $2(11,1)$ \\
& 2. Administrar medicamentos conforme prescrição médica & $1(5,5)$ \\
\hline
\end{tabular}


alteração, a fim de reduzir as complicações e melhorar a recuperação do paciente. Em estudo sobre a criação e implementação de um Enhanced recovery after surgery pathways, ferramenta implementada internacionalmente, com vistas a fornecer diretrizes para a tomada de decisão baseada em evidências, tendo como meta maior homogeneizar a assistência, sem comprometer a qualidade do atendimento em pacientes prostatectomizados radicalmente, identificou-se a associação entre essa ferramenta e a diminuição do tempo de internação hospitalar e das taxas de complicação pós-operatória e readmissões ${ }^{23}$. Nesse sentido, os profissionais responsáveis por identificar, prevenir e tratar as complicações relacionadas ao pós-operatório da prostatectomia deverão buscar ferramentas que subsidiem sua prática.

Estudo assevera que, para prevenir tal agravo, a enfermagem deve substituir, assim que possível, a solução de irrigação do cateter por uma solução salina normal, pois o excesso de irrigação durante o trans e o pós-operatório é uma das causas da hiponatremia ${ }^{22}$.

Portanto, a partir do exposto, a enfermagem deve realizar atividades voltadas às necessidades de cada paciente, proporcionando um cuidado holístico, considerando o indivíduo como um ser complexo, inserido em um contexto social particular.

\section{Conclusão}

Conclui-se que os profissionais de enfermagem enfatizaram as orientações no pós-operatório, o tratamento da incontinência urinária e da dor e o acompanhamento psicológico, estando algumas das atividades inseridas nesses cuidados em descompasso com a literatura.

Tal resultado demonstra o reduzido conhecimento desses profissionais em determinados aspectos mais específicos do cuidado ao pós-operatório do prostatectomizado. Tal constatação pode ser efeito da realidade encontrada neste estudo, no qual a maioria desses profissionais de enfermagem entrevistados não havia recebido treinamento no serviço de urologia. Assim, ressalta-se a importância de capacitação específica para o setor de urologia e de um maior conhecimento dos profissionais acerca das necessidades particularizadas da clientela atendida.

Destarte, é de suma relevância conhecer as atividades realizadas pela equipe de enfermagem para o paciente em pós-operatório de prostatectomia, com vistas a identificar possíveis falhas em sua execução, e, assim, promover a educação continuada de modo a proporcionar um aperfeiçoamento contínuo dos cuidados preconizados para essa clientela.

Entre as limitações do estudo destacaram-se a reduzida amostra e um único cenário, que impedem a generalização dos achados.

\section{REFERÊNCIAS}

1.Ministério da Saúde (Br). Instituto Nacional de Câncer (INCA). Estimativa 2012: incidência de câncer no Brasil. Brasília (DF): Ministério da Saúde; 2012.

2.Santos A, Gonçalves N, Santos MJ, Rolo F, Macedo A. Avaliação de práticas e conhecimentos dos homens relativamente a doença prostática, em Portugal - Estudo epidemiológico. Acta Urol Belg. 2007; 24(4):25-32.

3.Smeltzer SC, Bare BG, Hinkle JL, Cheever KH. Tratado de enfermagem médico cirúrgica. $12^{\mathrm{a}}$ ed. Rio de Janeiro: Guanabara Koogan; 2011.

4.Napoleão AA, Caldato VG, Petrilli Filho JF. Diagnósticos de enfermagem para o planejamento da alta de homens prostatectomizados: um estudo preliminar. Rev Eletr Enf. [internet] 2009 [citado em 19 set 2012]; 11(4):286-94. Disponível em: http://www.fen.ufg.br/ fen_revista/v11/n2/v11n2a08.htm.

5.Burt J, Caelli K, Moore K, Anderson M. Radical prostatectomy: men's experiences and postoperative needs. J Clin Nurs. [internet] 2005 [cited in 2012 Aug 12]; 14:883-90. Available from: http://onlinelibrary.wiley. $\mathrm{com} / \mathrm{doi} / 10.1111 / \mathrm{j} .1365-2702.2005 .01123 . \mathrm{x} / \mathrm{pdf}$.

6.Vianna MC, Napoleão AA. Reflexões sobre cuidados de enfermagem para a alta de pacientes prostatectomizados. Ciênc cuid saúde. 2009; 8:269-73.

7.Portes TA, Bernardo PLA, Faccio Júnior FN. Ressecção transuretral da próstata (RTUP): complicações transoperatórias e pós-operatórias em hospital universitário. Arqu Ciênc Saúde. 2004; 11:199-204.

8.Nunes P, Rolo F, Mota A. Prostatectomia radical e atividade sexual. Acta Urol. 2004; 21(1):15-31.

9.Santos DRF, Silva FBBL, Saldanha EA, Lira ALBC, Vitor AF. Cuidados de enfermagem ao paciente em pós-operatório de prostatectomia: revisão integrativa. Rev Eletr Enf. [internet] 2012 [citado em 19 set 2012]; 14(3):690-701. Disponível em: http://www.fen.ufg.br/ fen_revista/v14/n3/pdf/v14n3a27.pdf.

10. $\bar{N}$ apoleão AA, Carvalho EC. Aplicabilidade de intervenções prioritárias da NIC para o diagnóstico de enfermagem desobstrução ineficaz de vias aéreas. Cogitare enferm. 2007; 12(1):9-19.

11.Abraão SR, Bezerra ALQ, Branquinho NCSS, Paranaguá TTB. Caracterização, motivação e nível de satisfação dos técnicos de enfermagem de um hospital universitário. Rev enferm UERJ. 2010; 18:253-8.

12.Phillips C, Gray RE, Fitch MI, Labrecque M, Fergus K, Klotz L. Early postsurgery experience of prostate cancer patients and spouses. Cancer Pract. 2000; 8(4):165-70. 13.Mata LRF, Carvalho EC, Napoleão AA. Validação por peritos de intervenções de enfermagem para a alta de pacientes submetidos à prostatectomia. Texto \& contexto enferm. 2011; 20:36-44.

14.Saldanha EA, Fernandes MICD, Medeiros ABA, Frazão CMFQ, Costa IA, Lira ALBC. A teoria de Callista Roy, a NANDA-I e o cuidado ao paciente prostatectomizado. Rev enferm UERJ. 2012; 20:764-70.

15.Alves LAF, Santana RF, Schulz RS. Percepções de idosos sobre a utilização de fraldas durante a hospitali- 
zação. Rev enferm UERJ. 2014; 22:371-5.

16.Kim HS. Development and evaluation of self-care agency promoting programme for prostatectomy patients. J Urol Nurs. 2011; 5(1):34-44.

17. Honório MO, Santos SMA. A rede de apoio ao paciente incontinente: a busca por suporte e tratamentos. Rev enferm UERJ. 2010; 18:383-8.

18.Schover LR, Kaaij MVD, Dorst EV, Creutzberg C, Huyghe E, Kiserud CE. Sexual dysfunction and infertility as late effects of cancer treatment. Eur J Cancer. 2014; 12:41-53.

19. Mata LRF, Napoleão AA. Intervenções de enfermagem para alta de paciente prostatectomizado: revisão integrativa. Acta Paul Enferm. 2010; 23:574-9.

20.Cockle-Hearne J, Charnay-Sonnek F, Denis L, Fair- banks HE, Kelly D, Kav S. et al.The impact of supportive nursing care on the needs of men with prostate cancer: a study across seven European countries. Br J Cancer. 2013; 109:2121-30.

21.Mata LRF, Silva AC, Pereira MG, Carvalho EC. Telephone follow-up of patients after radical prostatectomy: a systematic review. Rev Latino-Am Enferm. 2014; 22:337-45.

22.Eaton J. Detection of hyponatremia in the PACU. J Perianesth Nurs. 2003; 18:392-7.

23.Abou-Haidar H, Abourbih S, Braganza D, Al Qaoud T, Lee L, Carli F. et al. Enhanced recovery pathway for radical prostatectomy: Implementation and evaluation in a universal healthcare system. Can Urol Assoc J. 2014; 8:11-2. 